\title{
Indirect comparison between ferric carboxymaltose and oral iron replacement in heart failure with reduced ejection fraction: a network meta-analysis
}

\author{
Edoardo Sciatti ${ }^{1}$, Ugo Nesti ${ }^{2}$, Andrea di Lenarda ${ }^{3}$ \\ ${ }^{1}$ Cardiology Unit, ASST del Garda, Hospital of Desenzano del Garda (BS); ${ }^{2}$ Biostatistician, Pencil and Papers, Pisa; \\ ${ }^{3}$ Cardiovascular Center, University Hospital and Health Services of Trieste, Italy
}

\begin{abstract}
Treatment of iron deficiency (ID) in patients with heart failure (HF) has improved symptoms, quality of life, exercise capacity and has reduced hospitalizations in randomized controlled trials (RCTs) and meta-analyses. Intravenous ferric carboxymaltose (FCM) provided convincing results in this field, while oral iron supplementation failed. However, FCM and oral iron were compared to placebo, and a comparison between the two strategies is still lacking. We aimed to fill this gap of knowledge with an indirect comparison between them by means of a network meta-analysis of RCTs. Five studies measuring exercise capacity (i.e., 6minute walking test) and quality of life (i.e. Kansas City Cardiomyopathy Questionnaire) were eligible to be included in our review. Given the limitations of a network meta-analysis, our findings support the better efficacy of FCM than oral iron as regards exercise capacity, with a trend towards an improvement in quality of life, suggesting that FCM seems to be strategy of choice to correct ID in HF patients.
\end{abstract}

Correspondence: Dr. Edoardo Sciatti, MD, Cardiology Unit, ASST del Garda, Hospital of Desenzano del Garda (BS), Italy.

Tel. +39.030.9145227.

E-mail: edoardo.sciatti@gmail.com

Key words: Ferinject; ferric carboxymaltose; quality of life; 6-minute walking test; heart failure.

Contributions: ES, drafted the paper; UN, performed the statistical analysis; ADL, designed the analysis and revised the draft.

Conflict of interest: The authors declare no conflict of interest.

Funding: The work was funded by Ethos s.r.1., via Berna 9, 00144 Rome, Italy

Received for publication: 26 November 2020.

Accepted for publication: 26 January 2021.

${ }^{\circ}$ Copyright: the Author(s), 2021

Licensee PAGEPress, Italy

Monaldi Archives for Chest Disease 2021; 91:1703

doi: 10.4081/monaldi.2021.1703

This article is distributed under the terms of the Creative Commons Attribution Noncommercial License (by-nc 4.0) which permits any noncommercial use, distribution, and reproduction in any medium, provided the original author(s) and source are credited.

\section{Introduction}

Recent advances in the management of heart failure (HF) with reduced ejection fraction (HFrEF) have changed the natural history of this syndrome and improved patients' outcomes [1]. However, their quality of life and functional capacity are still poor: fatigue and dyspnoea restrict the daily activity and contribute to high morbidity [2]. Several mechanisms unrelated to hemodynamic impairment may be responsible to a reduced exercise tolerance. In particular, comorbidities play a significant role in increasing the severity of symptoms, reducing tolerance to treatment, and determining a worse prognosis [2,3]. Among them, iron deficiency (ID), defined as serum ferritin level $<100$ $\mu \mathrm{g} / \mathrm{L}$ (absolute ID) or $100-299 \mu \mathrm{g} / \mathrm{L}$ with transferrin saturation < $20 \%$ (relative ID), affects nearly $40 \%$ of HF patients, independently from anemia [4]. Treatment of ID in patients with HF has improved symptoms, quality of life, exercise capacity and has reduced hospitalizations in randomized controlled trials (RCTs) and meta- analyses, independently from hemoglobin levels [510]. However, these studies were conducted comparing intravenous administration of ferric carobxymaltose (FCM) vs placebo on top of standard-of-care HF therapy, while only a large $\mathrm{RCT}$ investigated the efficacy of the inexpensive oral iron replacement vs placebo, founding a neutral effect on exercise capacity and quality of life [11]. According to these results, assessment of iron status with measurement of serum ferritin and transferrin saturation, to detect ID, and iron therapy with intravenous FCM in patients with HF and ID are now recommended in current guidelines to alleviate HF symptoms, and improve exercise capacity and quality of life [1]. However, to date no RCTs have directly compared FCM vs oral iron in the context of HFrEF. The aim of this paper is to fill this gap of knowledge with an indirect comparison between the two therapeutic strategies by means of a network meta-analysis.

\section{Materials and Methods}

The reporting of this review is consistent with the Preferred Reporting of Items for Systematic Reviews and Meta-Analyses (PRISMA) guidelines [12] as well as the Meta-Analysis of Observational Studies in Epidemiology (MOOSE) recommendations [13].

\section{Search strategy and inclusion of studies}

We searched PubMed, EMBASE, Web of Science, SCOPUS, Cochrane Central, clinicaltrials.gov as well as the preprint server 
Medrxiv, to obtain relevant articles on iron therapy in HFrEF, published till September 2020. A sensitive search was designed using keywords like 'Ferinject' OR 'ferric carboxymaltose' OR 'oral iron' AND 'heart failure'.

\section{Study design} RCTs.

Articles eligible for inclusion in our review were only

\section{Study population and exposures}

Studies measuring exercise capacity and quality of life were eligible to be included in our review.

\section{Study outcomes}

Outcomes included the six-minute walking test (6MWT) for exercise capacity and the Kansas City Cardiomyopathy Questionnaire (KCCQ) for quality of life.

\section{Exclusion criteria}

Studies published in non-English languages, RCTs without a comparator group, observational studies (retrospective cohorts, prospective cohorts, case-control studies, case-series), casereports and systematic and narrative reviews were not eligible for inclusion.

\section{Study selection}

Eligible studies were analysed for removal of duplicates. At level 1, titles and abstracts of all the retrieved articles were individually screened. Full-texts of studies eligible for inclusion were examined at level 2, and discrepancies were resolved through mutual consensus among the team members.

\section{Data extraction, assessment of quality and analysis}

Comparability of articles was based on the PICO criteria: the population, intervention, comparison, and outcome [14]. If the similarity assumption was not satisfied, the other two assumptions were negatively affected [15], and required check for the heterogeneity error $[16,17]$.

Inconsistency was tested at 2 levels $[18,19]$ : The first is a global approach to test for overall inconsistency, in which the level of inconsistency is computed according to the type of between treatment comparison for all cases and then the values are used to test for global linearity via the Wald test. The second is a local approach in which each treatment is individually examined (nodesplitting) and the outcomes of direct and indirect comparisons are statistically tested.

To establish the degree of efficacy of the treatments under study, we used the SUCRA method (surface under the cumulative ranking curve analysis). Network Forest Plots (NFP) were used to provide information on the effect size of each study and each treatment. Once the comparative effectiveness of the treatments was evaluated through the previous steps, the next step was to rank the treatments to identify superiority. In other words, treatment interventions showing the highest treatment effect were evaluated by an algorithm using the network rank and SUCRA to classify treatments. Meta-regression analysis was performed to examine the source of heterogeneity between studies and identify potential confounding covariates specifically for the impact of FCM treatment in patients with heart failure with reduced ejection fraction. All the statistical analysis were performed using STATA 14 software (StataCorp, 4905 Lakeway Drive, College Station, Texas 77845 USA).

\section{Risk of bias assessment}

In order to avoid publication bias [20] and relative large treatment effects [21] given by studies with small patient samples, we performed a Network Meta-Analysis (NMA) by means of the random effect model [22] and then by network funnel plot. Once the plots are generated, publication bias is visually inspected using the criterion of symmetry. Sensitivity analysis was performed when necessary.

\section{Results}

Our database search identified a total of 37 records. Of them, after removal of duplicates and assessment of eligibility only 5 were RCTs and were included in the analysis.

For the outcome 6MWT four trials were selected [5,6,10,11]. The network forest plot suggests that both iron formulations are more effective than placebo, with a greater effect coming from FCM (Figure 1). The interval plot highlights that FCM is better than placebo, but also than oral iron by means of an indirect comparison; moreover, placebo is more efficacious than oral iron (Figure 2). Finally, the SUCRA analysis confirms that FCM is the best treatment option (probability $55.3 \%$ ), with an area of 0.7 , while the oral iron has the higher probability to be the worst treatment $(68.8 \%)$, with an area of 0.3 (Table 1 ).

Regarding the outcome quality of life by KCCQ three trials were chosen $[5,6,11]$. The network forest plot suggests that both iron formulations are more effective than placebo, with a greater effect coming from FCM (Figure 3). The interval plot highlights that only FCM is better than placebo, with a trend toward more efficacy than oral iron by means of an indirect comparison (Figure 4). Finally, the SUCRA analysis confirms that FCM is the best treatment option (probability $76.4 \%$ ), with an area of 0.9 , while the oral iron has the higher probability to be the second treatment $(69.3 \%)$, with an area of 0.6 (Table 2 ).

Table 1. SUCRA analysis for the outcome 6MWT.

\begin{tabular}{lccc} 
Study and rank & FCM & $\begin{array}{c}\text { Treatment } \\
\text { Oral iron }\end{array}$ & Placebo \\
Best & 55.3 & 19.1 & 25.6 \\
$2^{\text {nd }}$ & 29.5 & 13.1 & 57.4 \\
\hline Worst & 15.2 & 67.8 & 17.0 \\
Mean rank & 1.6 & 2.5 & 1.9 \\
\hline SUCRA & 0.7 & 0.3 & 0.5 \\
\hline
\end{tabular}

Table 2. SUCRA analysis for the outcome KCCQ.

\begin{tabular}{lccc} 
Study and rank & FCM & $\begin{array}{c}\text { Treatment } \\
\text { Oral iron }\end{array}$ & Placebo \\
Best & 76.4 & 23.6 & 0.0 \\
$2^{\text {nd }}$ & 23.6 & 69.3 & 7.1 \\
\hline Worst & 0 & 7.1 & 92.9 \\
Mean rank & 1.2 & 1.8 & 2.9 \\
\hline SUCRA & 0.9 & 0.6 & 0.0 \\
\hline
\end{tabular}




\section{Discussion}

To the best of our knowledge, this is the first report about the (indirect) comparison between FCM and oral iron as regards functional capacity and quality of life in patients with HFrEF. Given the limitations of a NMA, our findings support the better efficacy of FCM than oral iron as regards the 6MWT, with a trend towards an improvement in quality of life.

In addition to its well-known effects on hemoglobin levels and oxygen transport to the tissues, iron directly affects tissue oxidative capacity, energy metabolism and mitochondrial function in all tissues, including the skeletal muscles and the myocardium [2325]. This explains why ID is related to HF symptoms and patients' activity. Moreover, multiple studies have shown that ID is an inde- pendent determinant of death or hospitalizations in patients with chronic or acute HF [4,26,27], suggesting that it should be searched and corrected in every subject. However, the correction of ID is challenging in HF patients. The only large trial analyzing the efficacy of high-dose oral iron (i.e., iron polysaccharide) in this context, namely the Iron Repletion Effects on Oxygen Uptake in Heart Failure (IRONOUT HF) trial, was neutral after 16 weeks of treatment [11]. This happened because oral iron therapy produced a minimal improvement in iron stores, even if the dose administered was 15 -fold higher than that of FCM in the FAIR-HF trial $[5,11]$. This suggests that the route of administration, rather than the strategy, was lacking clinical benefit [11]. On the other hand, the CONFIRM-HF trial demonstrated a $61 \%$ relative decrease in HF hospitalization for worsening HF and an improvement in functional capacity, symptoms, and quality of life in patients receiving

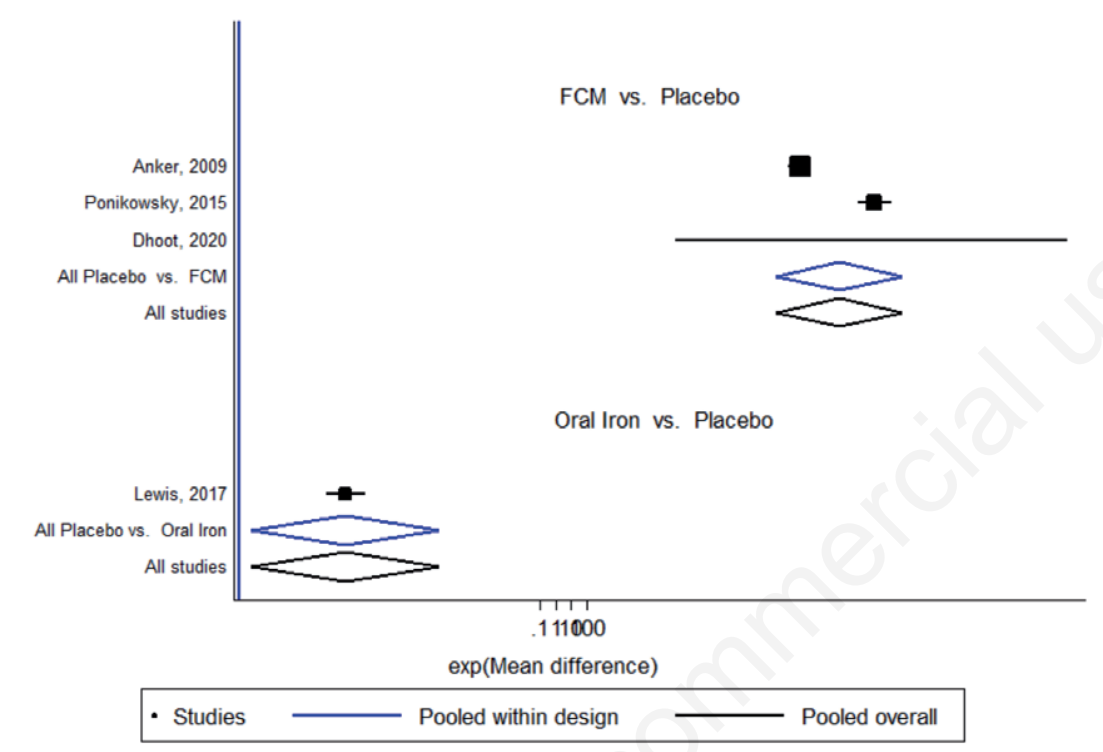

Test of consistency: $\operatorname{chi} 2(1)=19.15, \mathrm{P}=0.544$

Figure 1. Network forest plot for 6MWT.

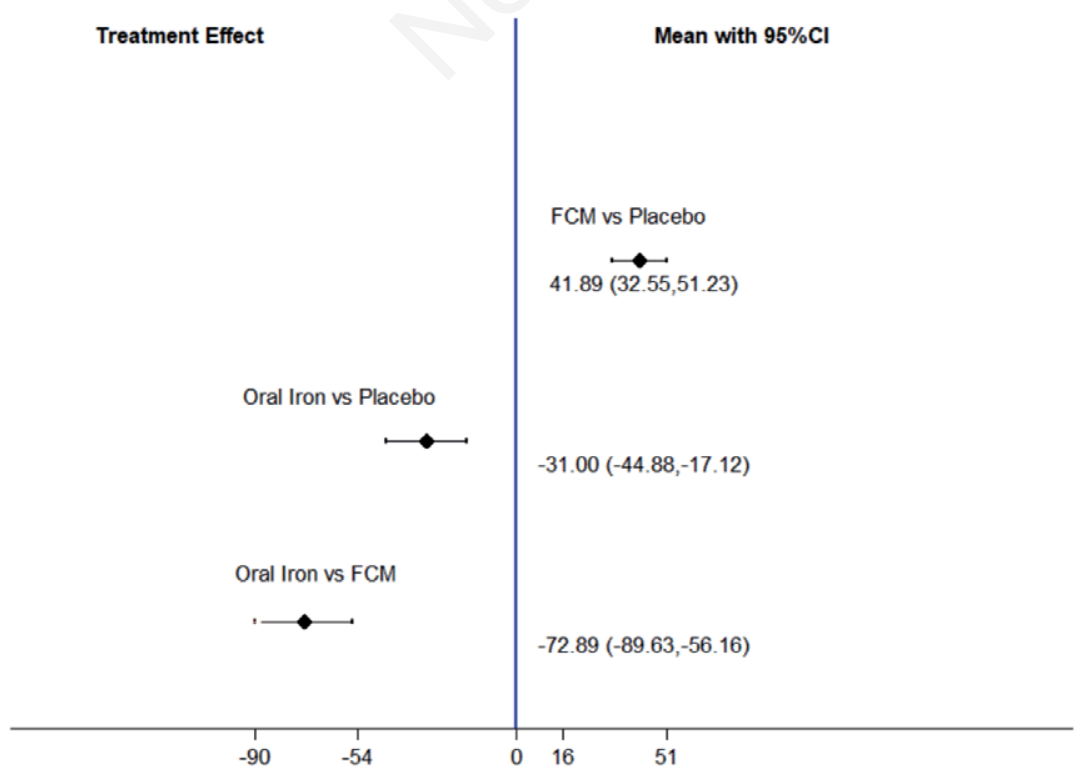

Figure 2. Interval pot for 6MWT. 
intravenous FCM [6]. A meta-analysis of five randomized clinical trials with intravenous iron supplementation (either iron sucrose and FCM) described a reduction of the combined endpoint of allcause death or cardiovascular (CV) hospitalization (OR 0.44, 95\%CI $0.30-0.64, \mathrm{p}<0.0001)$, CV death or hospitalization for worsening $\mathrm{HF}$ (OR $0.39,95 \% \mathrm{CI} 0.24-0.63, \mathrm{p}=0.0001$ ), and $\mathrm{HF}$ hospitalization (OR $0.28,95 \%$ CI $0.16-0.50, p<0.0001$ ), in parallel with an improvement of quality of life, functional capacity and other symptoms [7]. Notably, the results were mainly driven by the two trials with FCM (i.e., FAIR-HF and CONFIRM-HF) [5,6].

Recently, the "Study to Compare Ferric Carboxymaltose with Placebo in Patients with Acute Heart Failure and Iron Deficiency (AFFIRM-AHF)" enrolled 1132 patients with a left ventricular ejection fraction $<50 \%$ and ID, stabilized after an episode of acute HF. Treatment with FCM for 52 weeks was safe and reduced the risk of HF hospitalizations, with no apparent effect on the risk of cardiovascular death [28].

Besides this suggestion, it is to be acknowledged that an excessive iron supplementation may lead to tissue deposition and to free radical tissue damage, so mortality-driven studies about intravenous iron are still needed to assess long-term safety. Furthermore, trials aimed at the assessment of the effects of intravenous iron therapy on mortality in patients with HFrEF are ongoing. These include the intravenous iron in patients with systolic HF and ID to improve morbidity and mortality (FAIR-HF2) using FCM, the randomized placebo-controlled trial of ferric carboximaltose as treatment for HF with ID (HEART-FID), and the Intravenous Iron Treatment in Patients with Heart Failure and Iron Deficiency (IRONMAN) using iron (III) isomaltoside (NCT02937454, NCT03036462, NCT03037931, NCT02642562, respectively). They will definitely demonstrate if

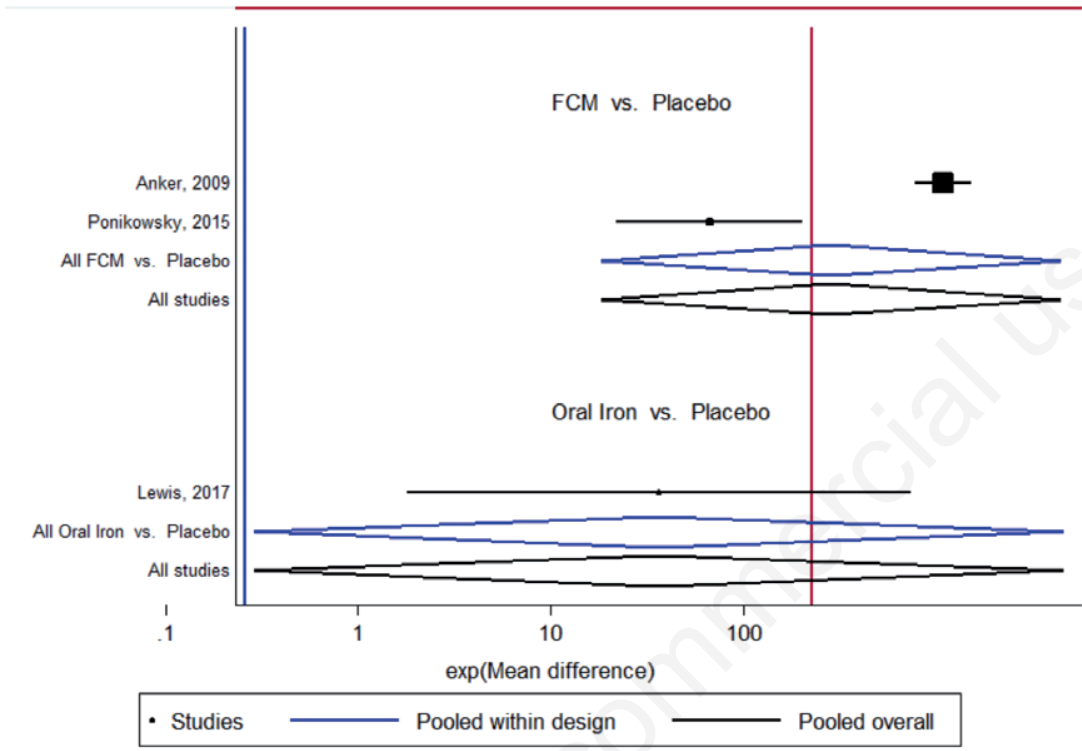

Test of consistency: $\operatorname{chi} 2(1)=2.12, \mathrm{P}=0.145$
Figure 3. Network forest plot for KCCQ.

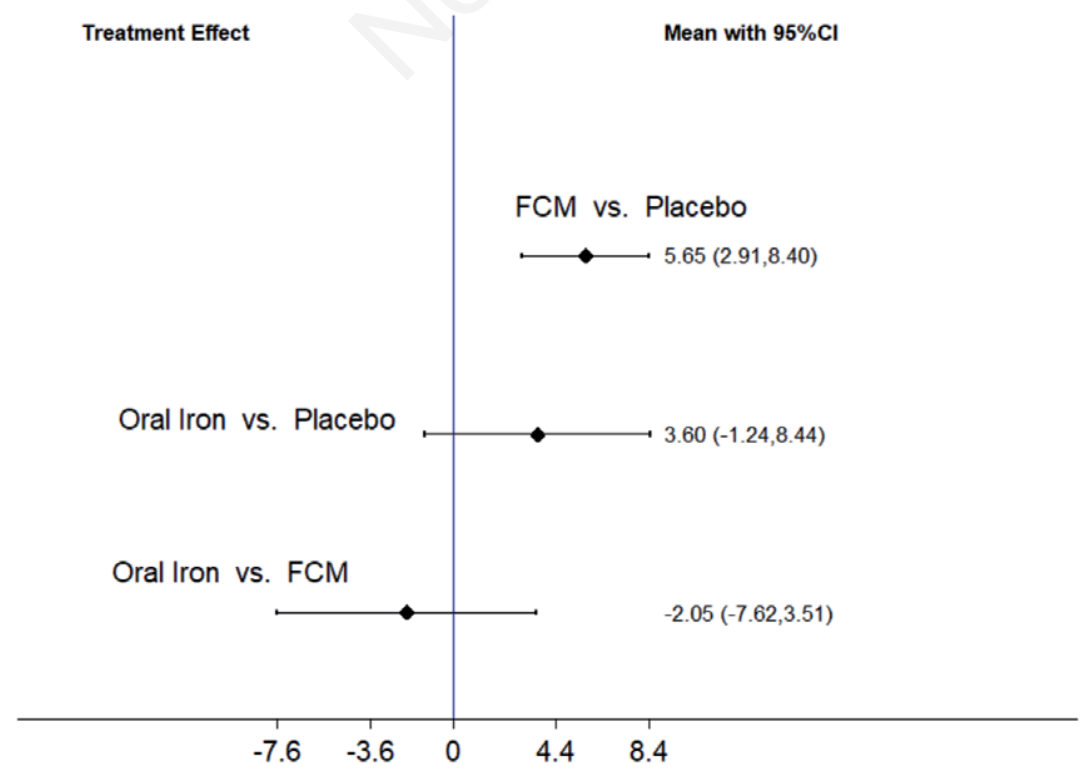

Figure 4. Interval pot for KCCQ. 
the correction of ID (principally with FCM) in HFrEF patients has an impact on hard clinical outcomes, like mortality. On the contrary a single RCT about oral iron replacement have been conducted to date [11]. We can also hypothesize that high-dose iron polysaccharide could not be the ideal compound as cited above, but we do not know if other oral formulations can be ineffective as well in terms of absorption and bioavailability. Given the easiness of oral administration than the organizational burden on the basis of FCM therapy, it could be worth conducting further multicenter RCTs about other types of oral iron in HFrEF, even if the physiology does not seem to suggest a possibility.

However, the present study support and reinforce the better efficacy of FCM than oral iron as regards exercise capacity and possibly quality of life, suggesting that FCM seems to be the strategy of choice to correct ID in HF patients. Nevertheless, the present study has some limitations. First, the small number of scientific articles used. Indeed, we only considered FCM as iv iron therapy since the bioequivalence among different iv formulations is not true due to the nanotechnology on the basis of these drugs [29,30]; moreover, FCM only is recommended by ESC guidelines [1]. Second, some articles have a small sample size that can not only cause publication bias [20], but also generate a relatively large treatment effect [21]. Another limitation is that the considered oral iron data refer to only one formulation [11]: we do not know whether other products with different absorption and bioavailability will be able to demonstrate different results, but at this moment this is the only RCT available.

To conclude, FCM seems to be strategy of choice to correct ID in HFrEF patients, with an evident better improvement in exercise capacity and quality of life than oral iron supplementation.

\section{References}

1. Ponikowski P, Voors AA, Anker SD, et al. 2016 ESC Guidelines for the diagnosis and treatment of acute and chronic heart failure: The Task Force for the diagnosis and treatment of acute and chronic heart failure of the European Society of Cardiology (ESC). Developed with the special contribution of the Heart Failure Association (HFA) of the ESC. Eur J Heart Fail 2016;18:891-975.

2. Metra M, Teerlink JR. Heart failure. Lancet 2017;390:1981-95.

3. van Deursen VM, Urso R, Laroche C, et al. Co-morbidities in patients with heart failure: an analysis of the European Heart Failure Pilot Survey. Eur J Heart Fail 2014;16:103-11.

4. Jankowska EA, Rozentryt P, Witkowska A, et al. Iron deficiency: an ominous sign in patients with systolic chronic heart failure. Eur Heart J 2010;31:1872-80.

5. Anker SD, Comin Colet J, Filippatos G, et al. Ferric carboxymaltose in patients with heart failure and iron deficiency. $\mathrm{N}$ Engl J Med. 2009;361:2436-48.

6. Ponikowski P, van Veldhuisen DJ, Comin-Colet J, et al. Beneficial effects of long-term intravenous iron therapy with ferric carboxymaltose in patients with symptomatic heart failure and iron deficiency. Eur Heart J 2015;36:657-68.

7. Jankowska EA, Tkaczyszyn M, Suchocki T, et al. Effects of intravenous iron therapy in iron-deficient patients with systolic heart failure: a meta-analysis of randomized controlled trials. Eur J Heart Fail 2016;18:786-95.

8. Anker SD, Kirwan BA, van Veldhuisen DJ, et al. Effects of ferric carboxymaltose on hospitalisations and mortality rates in iron-deficient heart failure patients: an individual patient data meta-analysis. Eur J Heart Fail 2018;20:125-33.

9. van Veldhuisen DJ, Ponikowski P, van der Meer P, et al. Effect of ferric carboxymaltose on exercise capacity in patients with chronic heart failure and iron deficiency. Circulation 2017;136:1374-83.

10. Dhoot S, Mittal S, Singh SP, et al. Effect of ferric-carboxy maltose on oxygen kinetics and functional status in heart failure patients with iron deficiency. Future Sci OA 2020;6:FSO467.

11. Lewis GD, Malhotra R, Hernandez AF, et al. Effect of oral iron repletion on exercise capacity in patients with heart failure with reduced ejection fraction and iron deficiency: The IRONOUT HF randomized clinical trial. JAMA 2017;317:1958-66.

12. Moher D, Liberati A, Tetzlaff J, et al. Preferred reporting items for systematic reviews and meta-analyses: The PRISMA Statement. PLoS Med 2009;6:e1000097.

13. Equator Network [Internet]. Meta-analysis of observational studies in epidemiology: a proposal for reporting. Meta-analysis of Observational Studies in Epidemiology (MOOSE) group. Accessed: 2020 Oct 20. Available from: https://www.equator- network.org/reporting-guidelines/metaanalysis-of-observational-studies-in- epidemiology-a-proposal-for-reporting-meta-analysis-of-observational-studies-inepidemiology-moose-group/

14. Reken S, Sturtz S, Kiefer C, et al. Assumptions of mixed treatment comparisons in health technology assessments: challenges and possible steps for practical application. PLoS One 2016;11:e0160712.

15. Jansen JP, Naci H. Is network meta-analysis as valid as standard pairwise meta-analysis? It all depends on the distribution of effect modifiers. BMC Med 2013;11:159.

16. Salanti G. Indirect and mixed-treatment comparison, network, or multiple-treatments meta-analysis: many names, many benefits, many concerns for the next generation evidence synthesis tool. Res Synth Methods 2012;3:80-97.

17. Veroniki AA, Vasiliadis HS, Higgins JP, Salanti G. Evaluation of inconsistency in networks of interventions. Int J Epidemiol 2013;42:332-45.

18. Tonin FS, Rotta I, Mendes AM, Pontarolo R. Network metaanalysis: a technique to gather evidence from direct and indirect comparisons. Pharm Pract (Granada) 2017;15:943.

19. Ioannidis JP, Cappelleri JC, Lau J. Issues in comparisons between meta-analyses and large trials. JAMA 1998;279:1089-93.

20. White IR. Network meta-analysis. Stata J 2015;15:951-85.

21. Pereira TV, Horwitz RI, Ioannidis JP. Empirical evaluation of very large treatment effects of medical interventions. JAMA 2012;308:1676-84.

22. Jansen JP, Fleurence R, Devine B, et al. Interpreting indirect treatment comparisons and network meta-analysis for healthcare decision making: report of the ISPOR Task Force on Indirect Treatment Comparisons Good Research Practices: part 1. Value Health 2011;14:417-28.

23. Jankowska EA, Malyszko J, Ardehali H, et al. Iron status in patients with chronic heart failure. Eur Heart J 2013;34:827-34.

24. Melenovsky V, Petrak J, Mracek T, et al. Myocardial iron content and mitochondrial function in human heart failure: a direct tissue analysis. Eur J Heart Fail 2017;19:522-30.

25. Stugiewicz M, Tkaczyszyn M, Kasztura M, et al. The influence of iron deficiency on the functioning of skeletal muscles: experimental evidence and clinical implications. Eur J Heart Fail 2016;18:762-73.

26. von Haehling S, Jankowska EA, van Veldhuisen DJ, et al. Iron deficiency and cardiovascular disease. Nat Rev Cardiol 2015;12:659-69. 
27. Núñez J, Comín-Colet J, Miñana G, et al. Iron deficiency and risk of early readmission following a hospitalization for acute heart failure. Eur J Heart Fail 2016;18:798-802.

28. Ponikowski P, Kirwan B-A, Anker SD, et al. Ferric carboxymaltose for iron deficiency at discharge after acute heart failure: a multicentre, double-blind, randomised, controlled trial. Lancet 2020;396:1895-904.

29. European Medicines Agency [Internet]. Reflection paper on the data requirements for intravenous iron-based nano-colloidal products developed with reference to an innovator medicinal product. Available from: https://www.ema.europa.eu/en/documents/scientific- guideline/reflection-paper-data-requirementsintravenous-iron-based-nano-colloidal-products-developed en.pdf

30. Martin-Malo A, Borchard G, Flühmann B, et al. Differences between intravenous iron products: focus on treatment of iron deficiency in chronic heart failure patients. ESC Heart Fail 2019;6:241-53. 